Pacific Journal of Mathematics

IRREDUCIBLE GROUPS OF AUTOMORPHISMS OF ABELIAN 


\title{
IRREDUCIBLE GROUPS OF AUTOMORPHISMS OF ABELIAN GROUPS
}

\author{
REINHOLD BAER
}

The group $\Gamma$ of automorphisms of the abelian group $A$ is termed irreducible, if 0 and $A$ are the only $\Gamma$-admissible subgroups of $A$. It is our aim to investigate the influence of the structure of the abstract group $\Gamma$ upon the structure of the pair $A, \Gamma$. In this respect we succeed in proving the following results:

If $\Gamma$ is locally finite, then $A$ is an elementary abelian $p$-group and the centralizer $\Delta$ of $\Gamma$ within the ring of endomorphisms of $A$ is a commutative, absolutely algebraic field of characteristic $p$. If we impose the stronger hypothesis that $\Gamma$ possesses an abelian torsion subgroup of finite index, then the rank of [the vector space] $A$ over $\Delta$ is finite and $\Gamma$ is a group of finite rank. If we add the further hypothesis that the orders of the elements in $\Gamma$ are bounded, then $A$ and $\Gamma$ are finite.

\section{Notations}

Locally finite group = group whose finitely generated subgroups are finite.

Almost abelian group = group possessing abelian subgroups of finite index

Group of finite rank = group whose finitely generated subgroups may be generated by fewer than a fixed number of elements

$\boldsymbol{m}$-group $\quad$ group by whose subgroups the minimum condition is satisfied.

Composition of the elements in the basic abelian group $A$ is denoted by addition. The effect of the endomorphism $\sigma$ of $A$ upon the element $a$ in $A$ will usually be denoted by $a \sigma$ unless $A$ is considered as a vector space over some field of scalars in which case the scalars may appear to the left of the vectors.

Proposition. If the irreducible group $\Gamma$ of automorphisms of the abelian group $A[\neq 0]$ is locally finite, then

(a) the centralizer $\Delta$ of $\Gamma$ [within the ring of endomorphisms of $A$ ] is a commutative, absolutely algebraic field of characteristic $p$, a prime, 
(b) $A$ is an elementary abelian p-group and

(c) the ring $\Lambda$ of endomorphisms, spanned by $\Gamma$, is locally finite.

Terminological Notes. The group $\Gamma$ of automorphisms of the abelian group $A[\neq 0]$ is irreducible, if no proper subgroup of $A$ is $\Gamma$-admissible.-The ring $A$ of endomorphisms, spanned by $\Gamma$, consists of all the endomorphisms of the form $\sum_{i} c_{i} \sigma_{i}$ with integral $c_{i}$ and $\sigma_{i}$ in $\Gamma$.- $A$ group [ring] is locally finite, if its finite subsets are contained in finite subgroups [subrings].

Proof. It is an immediate consequence of Schur's Lemma-see e.g. Jacobson [p. 26, Theorem 2]-that

(1) the centralizer $\Delta$ of $\Gamma$ is a [not necessarily commutative] field.

If $t$ is an element in $A$, then $t A$ is a $\Gamma$-admissible subgroup of $A$, since the ring $A$ of endomorphisms is spanned by $\Gamma$. Application of the irreducibility of $\Gamma$ shows that

(2) $t \Lambda=A$ for every $t \neq 0$ in $A$.

Consider now some element $\sigma$ in $\Delta$ and some element $t \neq 0$ in $A$. From (2) we deduce the existence of an element $\sigma^{\prime}$ in $\Lambda$ [depending on $\sigma$ and $t$ ] such that $t \sigma=t \sigma^{\prime}$. Since $A$ is centralized by $\Delta$, we have $\sigma \sigma^{\prime}=\sigma^{\prime} \sigma$; and it follows by complete induction that

$$
t \sigma^{i}=t \sigma^{i} \text { for every positive } i \text {. }
$$

Since $\Lambda$ is spanned by $\Gamma$, there exist [finitely many] automorphisms $\sigma_{i}$ in $\Gamma$ and integers $c_{i}$ such that $\sigma^{\prime}=\sum_{i=1}^{n} c_{i} \sigma_{i}$. The subgroup $\theta$ of $\Gamma$, generated by $\sigma_{1}, \cdots, \sigma_{n}$, is finite, since $\Gamma$ is locally finite. The subgroup $S=\{t \theta\}$ of $A$ is consequently finitely generated. By construction $S$ contains all the elements $t \sigma^{j}=t \sigma^{j}$; and the subgroup generated by them is as a subgroup of a finitely generated abelian group likewise finitely generated. Thus we have shown:

(3) If $t$ is an element in $A$ and $\sigma$ an element in $\Delta$, then the subgroup $\left\{t, t \sigma, \cdots, t \sigma^{i}, t \sigma^{i+1}, \cdots\right\}$ of $A$ is finitely generated.

Assume by way of contradiction that $A$ is torsionfree. Then [multiplication by] 2 is an element, not 0 , in the field $\Delta$ [by (1)] so that multiplication by $2^{-1}$ is likewise an automorphism of $A$ [which belongs to 4 ]. Application of (3) shows that for every $t \neq 0$ in $A$ the subgroup

$$
T=\left\{t, 2^{-1} t, \cdots, 2^{-i} t, \cdots\right\}
$$

is finitely generated and consequently a free abelian group, not 0 , of 
finite rank. But such a group is not closed under multiplication by $2^{-1}$. This is a contradiction showing that $A$ is not torsionfree. Conseequently there exists a prime $p$ such that $A$ contains elements of order $p$. The set of elements $x$ in $A$ with $p x=0$ is therefore a $\Gamma$-admissible subgroup, not 0 , of $A$; and we deduce from the irreducibility of $\Gamma$ that

(4) $p A=0$ for some prime $p$.

Thus (b) is proved.

If $t \neq 0$ is an element in $A$ and $\sigma \neq 0$ an element in $\Delta$, then $T=\left\{t, t \sigma, \cdots, t \sigma^{i}, t \sigma^{i+1}, \cdots\right\}$ is finitely generated by (3) and hence finite by (4). From $T \sigma \leqq T$ and (1) we deduce now that $\sigma \neq 0$ induces an automorphism of positive [finite] order $n$ in $T$. From $t\left(\sigma^{n}-1\right)=0$ and (1) we conclude that $\sigma^{n}=1$. Consequently

(5) there exists to every $\sigma$ in $\Delta$ a positive integer $k$ with $\sigma=\sigma^{k}$. Because of (1) and (5) we may apply a Theorem of Jacobson [p. 217, Theorem 1] showing that

(6) the field $\Delta$ is commutative.

From (4) we conclude that $p$ is the characteristic of $\Delta$; and it follows from (5) that $\Delta$ is absolutely algebraic. Thus we have verified (a).

If $\Xi$ is a finite subset of the ring $\Delta$, spanned by $\Gamma$, then there exists a finite subset $\Xi^{*}$ of $\Gamma$ such that every element in $\Xi$ has the form $\Sigma c(\sigma) \sigma$ for $\sigma$ in $\Xi^{*}$ and integral $c(\sigma)$. Since $\Gamma$ is locally finite, $\Xi^{*}$ is part of a finite subgroup $\theta$ of $\Gamma$. It is a consequence of (4) that the subring of $\Lambda$, spanned by $\theta$, is finite. Hence $\Xi$ is contained in a finite subring of $\Lambda$, proving (c).

REMARK 1. Assume that $\Delta$ is a commutative, absolutely algebraic field of prime number characteristic $p$ and that $V$ is a vector space over $\Delta$.

If firstly the rank of $V$ over $\Delta$ is finite, then it is well known and easily verified that the ring of linear transformations of $V$ over $\Delta$ [and its group of units] is locally finite.

We assume secondly the infinity of the rank of $V$ over $\Delta$. Denote by $A$ the ring of all linear transformations of $V$ over $\Delta$ and by $\Gamma$ the group of units in $\Lambda$ [= group of regular linear transformations in $\Lambda$ ]. It is obvious that $\Gamma$ is not even a torsion group so that we are quite far away from local finiteness. Cp. Corollary 1 below. We are going to construct various irreducible substructures of $\Lambda$ and $\Gamma$.

Denote by $\Lambda_{0}$ the totality of linear transformations $\sigma$ in $\Lambda$ with the property:

( 0 ) the subspace of vectors $v$ in $V$ with $v \sigma=0$ has finite co-rank in $V$. 
It is clear and well known that $A_{0}$ is an ideal in $A$, the minimal ideal, not 0 . Since $\Delta$ is a subring of $A$, we may form the sum $A_{0}+\Delta$ which is easily seen to be a locally finite subring of $\Lambda$. Its group of units is likewise locally finite and it is an irreducible group of automorphisms of the abelian group $V$.

If we denote by $I$ the subring of the integral multiples of 1 in $A$, then $I$ is the prime field of characteristic $p$. The sum $\Lambda_{0}+I$ is again a locally finite subring of $\Lambda$; and its group of units is likewise locally finite and an irreducible group of automorphisms of the abelian group $V$. In general, $A_{0}+I$ does not contain its centralizer 4 .

The preceding constructions show that such vector spaces $V$ over $\Delta$ always "arise" from locally finite irreducible groups of automorphisms of the abelian group $V$. On the other hand it is impossible to prove that an irreducible group of automorphisms of the abelian group $V$ which is contained in $\Lambda$ always contains a locally finite, irreducible group of automorphisms of $V$. This may be seen from the following construction:

the vector space $V$ over $\Delta$ is the direct sum $V=\sum_{\nu} S(\nu)$ of subspaces $S(\nu)$ of rank 1 . Their [infinite] cardinal is the rank of $V$ over 4 . Denote by $\theta$ the group of all [regular] linear transformations $\sigma$ in $\Gamma$ with the properties:

$$
\left\{\begin{array}{l}
S(\nu) \sigma=S(\nu) \text { for every } \nu \text { and } \\
\sigma \text { induces the identity in almost all } S(\nu)
\end{array} .\right.
$$

If we denote by $\theta(\nu)$ the subgroup of all those $\sigma$ in $\theta$ which induce the identity in every $S(\mu)$ with $\nu \neq \mu$, then it is easily seen that $\theta$ is the direct product of the $\theta(\nu)$ and that every $\theta(\nu)$ is isomorphic to the multiplicative group of $\Delta$ and hence an abelian torsion group of rank 1 without elements of order $p$.

The groups $\theta(\nu)$ and $\theta$ are equal to 1 if, and only if, $\Delta$ is the prime field of characteristic 2; and this possibility we exclude in the sequel.

Every infinite set possesses a torsionfree, simply transitive permutation group, as follows from the existence of torsionfree groups of any preassigned infinite cardinality. Consequently there exists a subgroup $\theta^{*}$ of $\Gamma$ which is torsionfree and induces faithfully a simply transitive group of permutations on the set of the $S(\nu)$. Naturally $\theta$ is normalized by $\theta^{*}$ so that the product $\theta \theta^{*}$ is a subgroup of $\Gamma$.

Every torsion subgroup of $\theta \theta^{*}$ is a subgroup of $\theta$ so that none of the torsion subgroups of $\theta \theta^{*}$ is an irreducible group of automorphisms of the abelian group $V$.

Consider a $\theta \theta^{*}$-admissible subgroup $T \neq 0$ of $V$. Then there exists an element $t \neq 0$ in $T$; and this element $t$ has the form 


$$
t=\sum_{\nu} t(\nu) \quad \text { with } t(\nu) \text { in } S(\nu)
$$

where almost all $t(\nu)$ are 0 . But $t \neq 0$ implies the existence of some $\mu$ with $t(\mu) \neq 0$. There exists a linear transformation $\sigma \neq 1$ in $\theta(\mu)$; and the element

$$
t-t \sigma=t(\mu)-t(\mu) \sigma
$$

is an element, not 0 , in $T \cap S(\mu)$. Consequently $[T \cap S(\mu)] \theta(\mu)=S(\mu)$ is part of $T$; and now it is clear that $T=T \theta \theta^{*}=V$. Thus $\theta \theta^{*}$ is in irreducible group of automorphisms, as we wanted to show.

LEMMA 1. The following properties of the group G are equivalent:

(i) $G$ is locally finite.

(ii) If the epimorphic image $H$ of $G$ is not locally finite, then there exists a minimal normal subgroup of $H$ and there exists a normal subgroup $N \neq 1$ of $H$ such that $H$ induces in $N$ a locally finite group of automorphisms.

(iii) Every epimorphic image $H \neq 1$ of $G$ possesses a locally finite normal subgroup $N \neq 1$.

(a) If $J$ is the intersection of all the normal subgroups $X$ of $G$ with locally finite $G / X$, then $G / J$ is locally finite.

(b) If the normal subgroup $X$ of $G$ with locally finite $G / X$ is itself not locally finite, then there exists a normal subgroup $Y$ of $G$ with $Y \subset X$ such that $X / Y$ is locally finite or nilpotent. (c) If an epimorphic image of $G$ is not locally finite, then it possesses a minimal normal subgroup.

Terminological Reminder. A group is nilpotent, if every epimorphic image, not 1 , has a center, not 1 .

Notes I. If the minimum condition is satisfied by the normal subgroups of $G$, then every epimorphic image, not 1 , of $G$ possesses a minimal normal subgroup. Furthermore there exists among the normal subgroups $X$ of $G$ with locally finite $G / X$ a minimal one, say $M$. If $K$ is a normal subgroup of $G$ with locally finite $G / K$, then $M \cap K$ is a normal subgroup of $G$ and $G /(M \cap K)$ is isomorphic to a subgroup of the direct product of the locally finite groups $G / M$ and $G / K$. Hence $G /(M \cap K)$ is likewise locally finite; and we deduce $M=M \cap K \subseteq K$ from the minimality of $M$. It follows that $M$ is the intersection $J$ of 
all the normal subgroups $X$ of $G$ with locally finite $G / X$, showing the local finiteness of $G / J$. Thus we have seen that in the presence of the minimum condition for normal subgroups of $G$ condition (iv.a) and the first half of condition (ii) may be omitted.

II. Dr. Karl Gruenberg (London) has pointed out to me that the group $G$ is-as a consequence of our Proposition-locally finite, if the minimum condition is satisfied by the normal subgroups of $G$ and if a finite term of the derived series of $G$ equals 1 . This fact is, by Note I, an obvious special case of Lemma 1: the equivalence of (i) and (iv). It was this suggestion of Dr. Gruenberg which led us to the present Lemma 1.

Proof. For future use in this proof we restate first two well known properties of local finiteness:

(1) An extension of a locally finite group by a locally finite group is a locally finite group.

(2) Products of locally finite normal subgroups are locally finite normal subgroups.

For the proofs of (1) and (2) see Specht [p. 141, Satz 40*].

Since epimorphic images of locally finite groups are locally finite, condition (ii) is an immediate consequence of (i). -Assume next the validity of (ii) and consider a homomorphic image $H \neq 1$ of $G$. We want to show then the existence of a locally finite normal subgroup, not 1 , of $H$. This is certainly the case, if $H$ itself is locally finite. Hence we assume next that $H$ is not locally finite. Then there exists, by (ii), a normal subgroup $N \neq 1$ of $H$ such that $H$ induces in $N$ a locally finite group of automorphisms. If $N$ happens to be locally finite, then we have again reached our goal; and thus we assume next that $N$ is not locally finite. There exist normal subgroups $X$ of $H$ with $N \cap X=1$; and among these there exists a maximal one, say $L$ [Maximum Principle of Set Theory]. Then $N \cap L=1$ so that $N L / L \cong N$. Hence $N L / L$ is a non locally finite normal subgroup of the epimorphic image $H / L$ of $H$ and $G$. Thus $H / L$ itself is not locally finite; and a second application of condition (ii) shows the existence of a minimal normal subgroup $K / L$ of $H / L$. From the maximality of $L$ we deduce that the normal subgroup $N \cap K$ of $H$ is different from 1. If $X$ is a normal subgorup of $H$ with $1 \subset X \subseteq N \cap K$, then $X \cap L=1$ so that $L \subset L X \subseteq K$. From the minimality of $K / L$ we deduce $K=L X$; and from Dedekind's modular law it follows that $N \cap K=X(N \cap K \cap L)=X$. Hence $N \cap K$ is a minimal normal subgroup of $H$. Denote by $C$ the centralizer of $N \cap K$ in $H$. Then $C$ is a normal subgroup of $H$ and $H / C$ is essentially the same as the group of automorphisms, induced in 
$N \cap K$ by $H$. This latter group is an epimorphic image of the group of automorphisms, induced in $N$ by $H$, which is locally finite. Thus $H / C$ is locally finite. If $N \cap K \cap C=1$, then $N \cap K$ is isomorphic to the subgroup $C(N \cap K) / C$ of the locally finite group $H / C$; and we have found a desired locally finite normal subgroup, not 1 , of $H$. If $N \cap K \cap C \neq 1$, then we deduce $N \cap K=N \cap K \cap C \subseteq C$ from the minimality of $N \cap K$; and this implies the commutativity of $N \cap K$. The minimality of the normal subgroup $N \cap K$ of $H$ is equivalent with the irreducibility of the group of automorphisms, induced in $N \cap K$ by $H$. Since $N \cap K$ is abelian, and since the induced irreducible group of automorphisms is locally finite, we may apply our Proposition (b). Thus $N \cap K$ is an elementary abelian p-group; and as such it is locally finite. This completes the derivation of (iii) from (ii).

Assume next the validity of (iii). Form the product $P$ of all the locally finite normal subgroups of $G$. This is by (2) a locally finite characteristic subgroup of $G$. If $P \neq G$, then we could deduce from (iii) the existence of a locally finite normal subgroup $Q / P \neq 1$ of $G / P$. Then $Q$ is an extension of the locally finite group $P$ by the locally finite group $Q / P$; and we deduce from (1) the local finiteness of $Q$. Hence $Q \subseteq P \subseteq Q$ by the construction of $P$ so that $P=Q$ and $Q / P=1$, a contradiction. Consequently $G=P$ is locally finite; and we have proved the equivalence of (i)-(iii).

If $G$ is locally finite, then the intersection $J$, occurring in (iv.a), is equal to 1 and $G / J=G$ is locally finite; and (iv.b) is satisfied because of the absence of normal subgroups of $G$ which are not locally finite. Likewise (iv.c) is satisfied by default because of the absence of epimorphic images which are not locally finite. Thus (iv) is a consequence of (i).

Assume finally the validity of (iv). Then $G / J$ is locally finite by (iv.a), if $J$ is the intersection of all normal subgroups $X$ with locally finite $G / X$. Assume by way of contradiction that $J$ is not locally finite. Then there exists by (iv.b) a normal subgroup $N$ of $G$ with $N \subset J$ such that $J / N$ is locally finite or nilpotent. If we let $G^{*}=G / N$ and $J^{*}=J / N$, then $J^{*}$ is a normal subgroup of $G^{*}$ with locally finite $G^{*} / J^{*}$ and $J^{*}$ is locally finite or nilpotent.

Assume first that $J^{*}$ is nilpotent. Since every epimorphic image of $G^{*}$ is an epimorphic image of $G$, we deduce from (iv.c):

(+) If an epimorphic image of $G^{*}$ is not locally finite, then it possesses a minimal normal subgroup.

Consider next an epimorphism $\sigma$ of $G^{*}$ upon some group which is not locally finite. Since $G^{*} / J^{*}$ is locally finite, $G^{* \sigma}$ is not an epimorphic image of $G^{*} / J^{*}$ so that $J^{* \sigma} \neq 1$. Since $J^{*}$ is nilpotent, so is $J^{* \sigma}$. It follows that the center $Z$ of $J^{* \sigma}$ is different from 1 . Since $Z$ is a 
characteristic subgroup of the normal subgroup $J^{* \sigma}$ of $G^{* \sigma}$, it is a normal subgroup of $G^{* \sigma}$. Since $Z$ is centralized by $J^{* \sigma}$, the group of automorphisms, induced in $Z$ by $G^{* \sigma}$, is an epimorphic image of $G^{* \sigma} / J^{* \sigma}$ and hence of the locally finite group $G^{*} / J^{*}$. Thus we have shown:

$(++)$ If an epimorphic image of $G^{*}$ is not locally finite, then it possesses a normal subgroup, not 1 , in which it induces a locally finite group of automophisms.

Combining $(+)$ and $(++)$ we see that condition (ii) is satisfied by $G^{*}$. Hence $G^{*}=G / N$ is locally finite. Consequently $N \subset J \subseteq N$ by the definition of $J$, a contradiction showing that $J / N$ is locally finite. But then $G / N$ is an extension of the locally finite group $J / N$ by the locally finite group $G / J$ so that $G / N$ is by (1) locally finite. Again we obtain the impossible $N \subset J \subseteq N$. This contradiction shows that $J$ is locally finite. Hence $G$ is, by (1), locally finite as an extension of the locally finite group $J$ by the locally finite group $G / J$. Thus (i) is a consequence of (iv) and we have shown the equivalence of conditions (i)-(iv).

As usual we say that a group is almost-abelian, if it possesses an abelian subgroup of finite index. The principal more or less well known properties of almost-abelian groups that we are going to need are collected in the following

Lemma 2. Assume that $G$ is an almost-abelian torsion group.

(a) $G$ is locally finite and possesses an abelian normal subgroup of finite index in $G$.

(b) Every abelian normal subgroup of finite index in $G$ is a product of finite abelian normal subgroups of $G$.

(c) $G$ is an m-group if, and only if, the minimum condition is satisfied by the normal subgroups of $G$.

Terminological Note. The group $G$ is an m-group, if the minimum condition is satisfied by the subgroups of $G$.

Proof. If $A$ is an abelian subgroup of finite index in $G$, then $A$ possesses but a finite number of conjugates in $G$, since the normalizer of $A$ contains $A$ and has therefore finite index in $A$. By Poincaré's Theorem the intersection $B$ of the subgroups conjugate to $A$ in $G$ has finite index too and is, naturally, an abelian normal subgroup of G.If $U$ is a finitely generated subgroup of $G$, then $U /(U \cap B) \cong U B / B$ is finite. It follows that $U \cap B$ is finitely generated; cp. e.g. Baer $[1 ;$ p. 396, (1.3)]. The finitely generated subgroup $U \cap B$ of the abelian 
torsion group $B$ is finite as is $U /(U \cap B)$ and hence $U$ is finite. This completes the proof of (a).

If $K$ is an abelian normal subgroup of $G$ with finite $G / K$, then $G$ induces in $K$ a finite group of automorphisms. Hence $t^{G}$ is, for every element $t$ in $K$, a finite class of conjugate elements. The finitely generated abelian torsion group $\left\{t^{G}\right\}$ is a finite abelian normal subgroup of $G$, proving (b).

Assume that the minimum condition is satisfied by the normal subgroups of $G$. There exists by (a) an abelian normal subgroup $N$ of $G$ with finite $G / N$. Thus $G$ induces in $N$ a group of automorphisms of finite order $n$. An immediate application of Baer [2; p. 4, Lemma 1] shows that $N$ is an $\boldsymbol{m}$-group. Since $G / N$ is finite and $N$ an $\boldsymbol{m}$-group, $G$ is an $m$-group, proving (c).

THEOREM. If the irreducible group $\Gamma$ of automorphisms of the [non-trivial] abelian group $A$ is an almost-abelian torsion group, then

(A) $A$ is an elementary abelian p-group,

(B) the centralizer $\Delta$ of $\Gamma$ [within the ring of endomorphisms of $A$ ] is a commutative, absolutely algebraic field of characteristic $p$,

(C) the rank of $A$ over $A$ is finite,

(D) $\Gamma$ is of finite rank.

Note on Terminology. The group $X$ is of finite rank, if there exists a positive integer $n$, the rank of $X$, such that every finitely generated subgroup of $X$ may be generated by $n$ [or fewer] elements.

Note on Hypotheses. It is a consequence of Lemma 2, (a) that $\Gamma$ is locally finite. But this hypothesis which sufficed for the Proposition is not sufficient under the present circumstances as may be seen from the following construction: Suppose that $A$ is a countably infinite, elementary abelian $p$-group. Denote by $\Gamma$ the set of all automorphisms $\sigma$ of $A$ with the property:

The subgroup of fixed elements of $\sigma$ has finite index in $A$.

It is easy to see that this set $\Gamma$ of automorphisms of $A$ is a locally finite group and that it is an irreducible group of automorphisms of $A$. The centralizer $A$ of $\Gamma$ [within the ring of endomorphisms of $A$ ] is the prime field of characteristic $p$ so that the rank of $A$ over $\Delta$ is infinite. Hence (C) does not hold and (D) does not hold either.

Proof. $\Gamma$ is by Lemma 2, (a) locally finite so that properties (A) and (B) are immediate consequences of the Proposition. Before effecting 
the general proof of $(\mathrm{C})$ we treat the following

Special Case. 4 is algebraically closed.

We may consider $A$ as a vector space over $\Delta$ and the $\Delta$-admissible subgroups of $A$ we may consequently term subspaces. If $U$ is a subset of $A$, then we denote by [U] the subspace $\sum_{u \in v} u \Delta$ of $A$ spanned by $U$.

Let $\theta$ be a finite abelian normal subgroup of $\Gamma$ and $\sigma$ a homomorphism of $\theta$ into the multiplicative group of roots of unity in 4 . Then we term the subspace $S$ of $A$ a $\sigma$-subspace [or more precisely a $\theta$ - $\sigma$-subspace] of $A$, if

$$
x \alpha=x \alpha^{\sigma} \text { for every } x \text { in } S \text { and every } \alpha \text { in } \theta .
$$

There exists an element $v \neq 0$ in $A$ and $v \theta$ is a finite subset of $A$, since $\theta$ is finite. Hence $[v \theta]$ is a subspace, not 0 , of $A$ which is of finite positive rank and $\theta$-admissible. Consequently there exists among the $\theta$-admissible subspaces of finite, positive rank one $R$ of minimal rank. In $R$ a finite abelian group $\theta^{*}$ of automorphisms [= linear transformations] is induced by $\theta$. From the minimality of the rank of $R$ we deduce

$$
R=[r \theta]=\left[r \theta^{*}\right] \text { for every } r \neq 0 \text { in } R .
$$

The ring $A$ of endomorphisms of $R$ which is spanned by $\theta^{*}$ and $A$ is commutative, since $\theta^{*}$ and $\Delta$ are commutative and centralize each other; and 0 and $R$ are the only $\Lambda$-admissible subgroups of $R$. Application of Schur's Lemma-cp. Jacobson [p. 26, Theorem 2]-shows that the centralizer $\Lambda^{*}$ of $\Lambda$ [within the ring of endomorphisms of $R$ ] is a field. From the commutativity of $\Lambda$ we deduce $\Lambda \subseteq \Lambda^{*}$. Hence $\Lambda$ is part of some commutative field of characteristic $p$. All the roots of unity of this field are already contained in the algebraically closed subfield $\Delta$. Since $\theta^{*}$ is finite and contained in $A$, the elements in $\theta^{*}$ are roots of unity and belong therefore to $\Delta$. If $\sigma$ is the homomorphism of $\theta$ which maps every element upon the automorphism it induces in $R$, then $\sigma$ is the epimorphism of $\theta$ upon $\theta^{*} \subseteq \Delta$ with $r \alpha=r \alpha^{\sigma}$ for every $r$ in $R$ and $\alpha$ in $\theta$. Thus $R$ is a $\sigma$-subspace of $A$; and from the minimality of the positive rank of $R$ we deduce that the rank of $R$ is 1 . Thus we have shown:

(1) If $\theta$ is a finite abelian normal subgroup of $\Gamma$, then there exists a $\sigma$-subspace of rank 1 of $A$ for some homomorphism $\sigma$ of $\theta$ into $A$.

If $\sigma$ is a homomorphism of the finite abelian normal subgroup $\theta$ of $\Gamma$ into $\Delta$, then there exist $\sigma$-subspaces of $A$ [like 0] and the sum $A(\sigma)=A(\theta, \sigma)$ of all the $\sigma$-subspaces of $A$ is again a $\sigma$-subspace of $A$. 
If $\gamma$ is an automorphism in $\Gamma$, then mapping the automorphism $\alpha$ in the finite abelian normal subgroup $\theta$ of $\Gamma$ upon $\gamma \alpha \gamma^{-1}=\alpha^{\gamma^{-1}}$ is an automorphism of $\theta$ and mapping the automorphism $\alpha$ in $\theta$ upon $\alpha^{\gamma^{-1} \sigma}$ is a homomorphism $\gamma^{-1} \sigma$ of $\theta$ in $A$. Since the numbers in $A$ commute with the automorphisms in $\Gamma$, we find that

$$
x \gamma \alpha=x \alpha^{\gamma^{-1}} \gamma=x\left(\alpha^{\gamma^{-1}}\right)^{\sigma} \gamma=x \gamma\left(\alpha^{\gamma^{-1}}\right)^{\sigma}=x \gamma \alpha^{\gamma^{-1} \sigma}
$$

for every $x$ in $A(\sigma)$ and $\alpha$ in $\theta$. This proves:

(2) If $\theta$ is a finite abelian normal subgroup of $\Gamma$, if $\sigma$ is a homomorphism of $\theta$ into $\Delta$ and $\gamma$ is an automorphism in $\Gamma$, then

$$
A(\sigma) \gamma=A\left(\gamma^{-1} \sigma\right) \text {. }
$$

Consider again a finite abelian normal subgroup $\theta$ of $\Gamma$ and a finite set $\Xi$ of homomorphisms $\sigma$ of $\theta$ into $\Delta$ with $A(\theta, \sigma) \neq 0$. If the sum of the $A(\sigma)$ with $\sigma$ in $\Xi$ were not their direct sum, then there would exist a minimal subset $\Xi^{\prime}$ of $\Xi$ such that the sum of the $A(\sigma)$ with $\sigma$ in $\Xi^{\prime}$ is not their direct sum. It is clear that $\Xi^{\prime}$ contains at least two homomorphisms. Hence we may number the homomorphisms in $\Xi^{\prime}$ as follows: $\sigma(0), \sigma(1), \cdots, \sigma(k)$ with $0<k$. Because of the minimality of $\Xi^{\prime}$ we have: $S=\sum_{i=1}^{k} A[\sigma(i)]$ is the direct sum of the $A[\sigma(i)]$, but $\sum_{i=0}^{k} A[\sigma(i)]$ is not. Hence

$$
A[\sigma(0)] \cap S \neq 0 .
$$

Consequently there exists an element $s \neq 0$ in $A[\sigma(0)] \cap S$. Clearly

$$
s=\sum_{i=1}^{k} s_{i} \quad \text { with } s_{i} \text { in } A[\sigma(i)] .
$$

If some $s_{i}$ were 0 , then $s$ would belong to $A[\sigma(0)] \cap \sum_{j \neq i} A[\sigma(j)]$ so that this intersection were not 0 and the sum of the $A[\sigma(j)]$ with $j \neq i$ were not their direct sum contradicting the minimality of $\Xi^{\prime}$. Thus $s_{i} \neq 0$ for $i=1, \cdots, k$. If $\alpha$ is an automorphism in $\theta$, then

$$
\sum_{i=1}^{k}\left(s_{i} \alpha^{\sigma(0)}\right)=s \alpha^{\sigma(0)}=s \alpha=\sum_{i=1}^{k}\left(s_{i} \alpha\right)=\sum_{i=1}^{k}\left(s_{i} \alpha^{\sigma(i)}\right) .
$$

Since $S$ is the direct sum of the $A[\sigma(i)]$ with $i=1, \cdots, k$, we deduce $s_{i} \alpha^{\sigma(0)}=s_{i} \alpha^{\sigma(i)}$ for $i=1, \cdots, k$ from this equation. Since every $s_{i} \neq 0$ and every $\alpha^{\sigma}$ is a number in the field, $\Delta$, we conclude $\alpha^{\sigma(0)}=\alpha^{\sigma(i)}$ for every $i$. Since this last equation is true for every $\alpha$ in $\theta$, we have shown $\sigma(0)=\sigma(i)$ for $i=1, \cdots, k$; and this is impossible. Thus we have shown that the sum of the $A(\sigma)$ with $\sigma$ in $\Xi$ is their direct sum.

Let now $B$ be the sum of all the $A(\sigma) \neq 0$. Then the result of the preceding paragraph of our proof shows that $B$ is the direct sum 
of the $A(\sigma)$, If $\gamma$ is an automorphism in $\Gamma$, then we deduce $B=B \gamma$ from (2). Since $B \neq 0$ is therefore a $\Gamma$-admissible subspace of $A$, and since $\Gamma$ is an irreducible group of automorphisms, we have $B=A$. If finally $n$ is the exponent of $\theta$ [so that $\theta^{n}=1$ ], then $\theta$ is mapped by every homomorphism $\sigma$ of $\theta$ into $\Delta$ into the finite group of $n$th roots of unity in the field $\Delta$. Hence there exists only a finite number of homomorphisms of $\theta$ into $\Delta$. We summarize these results as follows:

(3) If $\theta$ is a finite abelian normal subgroup of $\Gamma$, then $A$ is the direct sum of the finitely many $A(\theta, \sigma) \neq 0$ [with $\sigma$ a homomorphism of $\theta$ into 4$]$.

Next we recall that $\Gamma$ is an almost-abelian torsion group. Application of Lemma 2 shows the existence of an abelian normal subgroup $\Lambda$ of $\Gamma$ with finite $\Gamma / \Lambda$; and $\Lambda$ is the product of finite normal subgroups of $\Gamma$. Let $h=[\Gamma: \Lambda]$.

Consider next a finite abelian normal subgroup $\theta$ of $\Gamma$ with $\theta \subseteq \Lambda$. If $\lambda$ is an automorphism in $\Lambda$, then $\lambda$ induces the identity automorphism in $\theta$ [since $A$ is abelian]. If $\sigma$ is a homomorphism of $\theta$ into $A$, then $\sigma=\lambda \sigma$; and consequently there exist at most $h$ distinct homomorphisms of the form $\gamma \sigma$ for $\gamma$ in $\Gamma$. Assume now that the homomorphism $\sigma$ of $\theta$ into $\Delta$ has the additional property $A(\sigma) \neq 0$. Denote the distinct homomorphisms of the form $\gamma \sigma$ for $\gamma$ in $\Gamma$ by $\sigma(1), \cdots, \sigma(k)$. Then $k \leqq h$. The subspace $S=\sum_{i=1}^{k} A[\sigma(i)]$ is different from 0 ; and $S$ is $\Gamma$-admissible because of (2). Application of the irreducibility of $\Gamma$ shows $S=A$. From (3) we deduce now that $S$ is the direct sum of the $A[\sigma(i)]$ and that to every homomorphism $\sigma^{\prime}$ of $\theta$ into $\Delta$ with $A\left(\sigma^{\prime}\right) \neq 0$ there exists an $i$ with $\sigma^{\prime}=\sigma(i)$. If we say now that $\sigma$ is a relevant. homomorphism of $\theta$, if $\sigma$ is a homomorphism of $\theta$ into $\Delta$ with $A(\theta, \sigma) \neq 0$, then we may express our results as follows:

(4) If the finite abelian normal subgroup $\theta$ of $\Gamma$ is part of $A$, then there exist at most $h$ relevant homomorphisms $\sigma$ of $\theta$; and if $\sigma^{\prime}, \sigma^{\prime \prime}$ are relevant homomorphisms, then there exists an automorphism $\gamma$ in $\Gamma$ with $\sigma^{\prime}=\gamma \sigma^{\prime \prime}$.

Because of (4) there exists among the finite abelian normal subgroups of $\Gamma$ which are contained in $\Lambda$ one, say $A^{*}$, with a maximum number of relevant homomorphisms.

Suppose now that $\theta$ is a finite abelian normal subgroup of $\Gamma$ with $\Lambda^{*} \subseteq \theta \subseteq \Lambda$. If $\sigma$ is a relevant homomorphism of $\theta$ and $\sigma^{*}$ is the restriction of $\sigma$ to $\Lambda^{*}$, then

$$
x \alpha^{\sigma *}=x \alpha^{\sigma}=x \alpha \text { for } x \text { in } A(\theta, \sigma) \text { and } \alpha \text { in } A^{*} \text {. }
$$

It follows that $\boldsymbol{A}(\theta, \sigma) \subseteq A\left(\Lambda^{*}, \sigma^{*}\right)$. Hence $\sigma^{*}$ is a relevant homomorphism of $A^{*}$. But $A$ is by (3) both the direct sum of all the $A(\theta, \sigma)$. 
with relevant $\sigma$ and all the $A\left(\Lambda^{*}, \tau\right)$ with relevant $\tau$. This implies in particular that $A\left(\Lambda^{*}, \tau\right)$ is for every relevant $\tau$ the direct sum of all the $A(\theta, \sigma)$ with relevant $\sigma$ such that $\sigma^{*}=\tau$, since we have $A(\theta, \sigma) \leqq A\left(\Lambda^{*}, \sigma^{*}\right)=A\left(\Lambda^{*}, \tau\right)$. Hence the mapping $\sigma \rightarrow \sigma^{*}$ is a single valued mapping of the set of all the relevant homomorphisms of $\theta$ upon the full set of all the relevant homomorphisms of $\Lambda^{*}$. Because of the maximality of the number of relevant homomorphisms of $\Lambda^{*}$ it follows that this mapping is actually one-to-one. This implies in particular that $A(\theta, \sigma)=A\left(\Lambda^{*}, \sigma^{*}\right)$ for every relevant homomorphism $\sigma$ of $\theta$. Thus we have shown the following facts:

(5) If $\sigma$ is a relevant homomorphism of $\Lambda^{*}$, if $\theta$ is a finite abelian normal subgroup of $\Gamma$ with $A^{*} \leqq \theta \leqq \Lambda$, then there exists one and only one relevant homomorphism $\sigma^{\prime}$ of $\theta$ with

$$
\begin{gathered}
A\left(A^{*}, \sigma\right)=A\left(\theta, \sigma^{\prime}\right) \text { and } \\
x \alpha^{\sigma}=x \alpha^{\sigma^{\prime}}=x \alpha \text { for every } x \text { in } A\left(\Lambda^{*}, \sigma\right) \text { and } \alpha \text { in } \Lambda^{*} .
\end{gathered}
$$

By (1) there exists at least one relevant homomorphism $\sigma$ of $\Lambda^{*}$. Let $S=A\left(\Lambda^{*}, \sigma\right)$. If $\lambda$ is an automorphism in $\Lambda$, then there exists a finite abelian normal subgroup $\theta$ of $\Gamma$ which contains $A^{*}$ and $\lambda$ and which in turn is contained in $\Lambda$, since $\Lambda$ is the product of finite abelian normal subgroups of $\Gamma$. By (5) there exists one and only one relevant homomorphism $\sigma^{\prime}$ of $\theta$ with $S=A\left(\theta, \sigma^{\prime}\right)$. It follows in particular that $S=S \lambda$ and that the automorphism induced in $S$ by $\lambda$ is just the multiplication by the number $\lambda^{\sigma^{\prime}}$ in $A$. Thus we have shown:

(6) $S=S \Lambda$ and the automorphisms induced in $S$ by elements in $\Lambda$ are multiplications by numbers in 4 .

If $t \neq 0$ is some element in $S$, then $T=t \Delta$ is a subspace of rank 1. By (6) we have $T=T \Lambda \subseteq S$. The automorphisms in $\Gamma$ map $T$ upon subspaces of $\operatorname{rank} 1$; and because of $T=T \Lambda$ and $[\Gamma: \Lambda]=h$ the number of these subspaces is finite and does not exceed $h$. The sum of these subspaces $T \gamma$ for $\gamma$ in $\Gamma$ is different from 0 and it is $\Gamma$-admissible. Because of the irreducibility of $\Gamma$ it is $A$; and thus we have shown that $A$ is the sum of finitely many subspaces of rank 1 . Since their number does not exceed $h$, we have shown:

(7) the rank of $A$ over $\Delta$ does not exceed $h$. By (7) we have proven (C) in the special case.

Reduction of the general case to the special case. We note first that $\Delta$ is by (B) a commutative, absolutely algebraic field of characteristic $p$. Let $\Delta^{*}$ be the algebraic closure of $\Delta$. Then there exists a vector 
space $V$ over $\Delta^{*}$ with the following properties:

(i) $A$ is a subgroup of $V$ and the subfield $\Delta$ of $\Delta^{*}$ operates on $A$ in the preassigned way.

(ii) The $\Delta^{*}$-vector space $V$ is spanned by its subset $A$.

(iii) There exists a group $\Gamma^{*}$ of $\Delta^{*}$-automorphisms of $V$ such that $A$ is $\Gamma^{*}$-admissible and mapping every automorphism in $\Gamma^{*}$ upon its restriction on $A$ effects an isomorphism of $\Gamma^{*}$ upon $\Gamma$.

Denote by $\Gamma^{* *}$ the group of automorphisms of the additive group $V$, generated by $\Gamma^{*}$ and the multiplications by elements, not 0 , in $\Delta^{*}$. The isomorphic groups $\Gamma$ and $\Gamma^{*}$ are almost-abelian torsion groups. Since $\Delta^{*}$ is an absolutely algebraic field of characteristic $p$, its multiplicative group is an abelian torsion group. Since $\Gamma^{*}$ and $\Delta^{*}$ centralize each other, $\Gamma^{* *}$ is an almost-abelian torsion group.

We don't claim that $\Gamma^{* *}$ is an irreducible group of automorphisms of $V$. Hence we consider the $\Gamma^{* *}$-admissible subgroups $X$ of $V$ [these are exactly the $\Gamma^{*}$-admissible $\Delta^{*}$-subspaces of $V$ ] which satisfy $A \cap X=0$. There exist such subgroups $X$ as for instance $X=0$. Application of the maximum principle of set theory shows that among these subgroups $X$ there exists a maximal one, say $M$. We may form the $\Delta^{*}$-vector space $V / M$ and $\Gamma^{* *}$ induces on $V / M$ a group of linear transformations $\Xi$ which, as an epimorphic image of $\Gamma^{* *}$, is an almost-abelian torsion group.

Suppose that $S / M$ is a $\Xi$-admissible subspace, not 0 , of $V / M$. Then $S$ is a $\Gamma^{* *}$-admissible subspace of $V$ with $M \subset S$. Because of the maximality of $M$ we have $S \cap A \neq 0$. Because of the irreducibility of $\Gamma$ the subgroup of $A$ which is spanned by the $\Gamma$-admissible subset $(S \cap A) \Gamma$ is $A$. It follows from (i) to (iii) that $V$ is spanned by the subset $(S \cap A) \Gamma^{* *}$. Hence $S=V$, proving that $\Xi$ is an irreducible group of automorphisms of $V / M$. Its centralizer contains $4^{*}$. Since $\Delta^{*}$ is algebraically closed, and since the centralizer of the irreducible, almost-abelian torsion group $\Xi$ of automorphisms is, by Lemma 2, (a) and the Proposition, absolutely algebraic, $\Delta^{*}$ is exactly the centralizer of $\Xi$ [within the ring of endomorphisms of $V / M$ ]. Application of the Special Case shows that the rank of $V / M$ over $\Delta^{*}$ is finite. From $M \cap A=0$ and (i)-(iii) we deduce that the ranks of $A$ over $\Delta$ and of $V / M$ over $\Delta^{*}$ are equal. Hence the rank of $A$ over $\Delta$ is finite, proving (C).

Since $\Delta$ and $A$ centralize each other, we may form the product $\phi$ of the abelian group $\Lambda$ and the multiplicative subgroup of the, by (B), commutative field $\Delta$. It is clear that $\phi$ is an abelian group of automorphisms of $A$. The group $A$ is a torsion group as a subgroup of the torsion group $\Gamma$. The field $\Delta$ is by (B) an absolutely algebraic field of prime characteristic so that its multiplicative group is a group of 
roots of unity and hence a torsion group. The abelian group $\phi$ is therefore a product of two torsion subgroups and hence is a torsion group. Next we note that a subgroup of $A$ is $\phi$-admissible if, and only if, it is a $A$-admissible $\Delta$ - subspace of $A$.

Since the rank of $A$ over $\Delta$ is, by (C), finite, and since $\phi$-admissible subgroups of $A$ are subspaces of $A$, there exists among the $\phi$-admissible subspaces, not 0 , of $A$ one $D$ of minimal positive rank. The abelian group $\phi$ of automorphisms of $A$ induces in $D$ an abelian group $\phi^{*}$ of automorphisms. If $X \neq 0$ is a $\phi^{*}$-admissible subgroup of $D$, then $X$ is $\phi$-admissible and hence a subspace of $A$. Because of the minimality of the rank of $D$, the subspaces $X$ and $D$ have the same rank; and this implies $X=D$ so that $\phi^{*}$ is an irreducible group of automorphisms of $D$. It is a consequence of Schur's Lemma-cp. Jacobson [p. 26, Theorem 2]-that the centralizer $\phi^{* *}$ of $\phi^{*}$ within the ring of endomorphisms of $D$ is a [not necessarily commutative] field. But $\phi^{*}$ is a subgroup of the center of $\phi^{* *}$ which is a commutative field. Furthermore $\phi^{*}$ is a torsion group as an epimorphic image of $\phi$. Thus we see that $\phi^{*}$ is a subgroup of the group of roots of unity of a commutative field and as such $\phi^{*}$ is an abelian torsion group of rank 1 .

Since $D \neq 0$ is $\phi$-admissible, $D$ is a $A$-admissible subspace of $A$. Since $\Gamma / A$ is finite, $D \Gamma$ is a finite set of subspaces of $A$. The sum $T=\sum_{\sigma \in \Gamma} D \sigma$ of these finitely many subspaces is a $\Gamma$-admissible subspace of $A$; and we deduce $T=A$ from the irreducibility of $\Gamma$. Denote by $D_{1}, \cdots, D_{f}$ the finitely many distinct subspaces of the form $D \sigma$ with $\sigma$ in $\Gamma$; and denote by $\Lambda_{i}$ the totality of automorphisms in $\Lambda$ which induce the identity automorphism in $D_{i}$. If [as we may assume without loss in generality] $D=D_{1}$, then $A / A_{1}$ is essentially the same as a subgroup of $\phi^{*}$ showing that $\Lambda / \Lambda_{1}$ is of rank 1. If $D_{i}=D_{1} \sigma$, then $\Lambda_{i}=\sigma^{-1} \Lambda_{1} \sigma$ proving the isomorphy $\Lambda / \Lambda_{i} \simeq \Lambda / \Lambda_{1}$. Hence all the $\Lambda / \Lambda_{i}$ are abelian torsion groups of rank 1 . An automorphism in $A$ belongs to $A_{1} \cap \cdots \cap A_{f}$ if, and only if, it induces the identity automorphism in all the $D \sigma$ with $\sigma$ in $\Gamma$ and hence in their sum $T=A$. Thus $\Lambda_{1} \cap \cdots \cap \Lambda_{f}=1$; and this shows that $\Lambda$ is isomorphic to a subgroup of the direct product of the $f$ isomorphic groups $A / A_{i}$ of rank 1 . Hence $A$ is an abelian group of rank not exceeding $f$. But $\Gamma / A$ is finite [and $\Gamma$ is locally finite by Lemma 2, (a)] proving that $\Gamma$ too is of finite rank.

REMARK 2. The reader should notice that throughout the proof of Properties (C) and (D) we have not fully used the requirement that $\Gamma$ be an irreducible group of automorphisms. All we used is the fact that $\Gamma$ is an irreducible group of linear transformations of $A$ over $A$.

REMARK 3. It is impossible to prove (D) in the stronger form: 
For let $K$ be the algebraically closed, absolutely algebraic field of characteristic a prime $p$. If we denote by $A$ the additive group of $K$, then every element, not 0 , in $K$ induces in $A$ an automorphism by multiplication. The group $A$ of these automorphisms of $A$ is clearly irreducible and an abelian torsion group of rank 1. But it is not an $\boldsymbol{m}$-group, since it contains elements of every positive order prime to $p$. For a similar construction see Duguid-McLain.

If $\Gamma$ is a group of automorphisms of the abelian group $A$, then a ring $A$ of endomorphisms of $A$ is spanned by $\Gamma$. It is clear that $\Gamma$ and $A$ are centralized by the same automorphisms of $A$ and that a subgroup of $A$ is $\Gamma$-admissible if, and only if, it is $A$-admissible. In particular $\Gamma$ is an irreducible group of automorphisms of $A$ if, and only if, $A$ is an irreducible ring of endomorphisms of $A$. Because of this relation between $\Gamma$ and $\Lambda$ it is possible to express our results more forcefully within the framework of the theory of endomorphism rings.

The following lemma puts a number of well known results in a form convenient for our applications.

LEMMA 3. The following properties of the vector space $V$ of rank $>1$ over the [not necessarily commutative] field $\triangle$ are equivalent:

(a) The rank of $V$ over $\Delta$ is finite.

(b) If $\Delta$ is the centralizer of the irreducible ring $A$ of endomorphisms of the abelian group $V$, then $A$ is the centralizer of $\Delta$.

(c) There exists a finite irreducible group of linear transformations of the vector space $V$ over 4 .

Notational Remark. The set $\Xi$ of endomorphisms of the abelian group $A$ is irreducible, if 0 and $A$ are the only $\Xi$-admissible subgroups of $A$; and the set $\Lambda$ of linear transformations of the vector space $V$ over $\Delta$ is irreducible, if 0 and $V$ are the only $A$-admissible subspaces of $V$.-Groups of linear transformations consist of automorphisms.

Proof. Assume first that the rank of $V$ over $\Delta$ be finite. If $\Delta$ is the centralizer of the irreducible ring $\Lambda$ of endomorphisms of the abelian group $V$, then we apply the Density Theorem of Jacobson [p. 28] to see that $A$ is the ring of all linear transformations of the vector space $V$ over $A$. Hence (b) is a consequence of (a).

Assume next that the rank of $V$ over $\Delta$ be infinite. Denote by $A$ the ring of all the linear transformations $\sigma$ of the vector space $V$ over $\Delta$ with the property:

The rank of the subspace $V \sigma$ of $V$ is finite. 
If $S$ is a subspace of $V$ of finite co-rank, then the transformations in $\Lambda$ annihilating $S$ induce in the vector space $V / S$ over $A$ the ring of all linear transformations. This implies in particular that $\Delta$ is the centralizer of $\Lambda$. If $v \neq 0$ is an element in $V$, then $v \Lambda=V$. Since the rank of $V$ over $\Delta$ is infinite, $A$ is not the ring of all linear transformations of the vector space $V$ over $\Delta$. Thus (b) is not satisfied by $V$ and $A$; and this shows that (a) is a consequence of (b), proving the equivalence of (a) and (b).

Assume again that the rank $n$ of $V$ over $\Delta$ be finite. Then there exists a finite basis $B$ of $V$ over $A$. We distinguish two cases.

Case 1. The characteristic of $\Delta$ is not 2 .

Denote by $\theta$ the set of all the linear transformations $\sigma$ of $V$ over $\Delta$ with the property:

$\sigma$ is an automorphism of $V$ which maps every element in $B$ upon an element of the form $\pm b$ for $b$ in $B$.

It is clear that $\theta$ is a group whose order is $2^{n}(n !)$. The ring of endomorphisms of $V$ which is spanned by $\theta$ contains for every pair of elements $x, y$ in $B$ one and only one linear transformation $\lambda$ of $V$ over $\Delta$ such that $x \lambda=y$ and $b \lambda=0$ for $b \neq x$ in $B$. These form a soc. system of matrix units over $A$, showing that $\theta$ is a finite irreducible group of linear transformations of the vector space $V$ over $A$.

Case 2. The characteristic of $\Delta$ is 2 .

Then $B$ generates a subgroup $C$ of the abelian group $V$ whose order is exactly $2^{n}$. Denote by $\theta$ the set of all the linear transformations $\sigma$ of the vector space $V$ over $\Delta$ with the property:

$\sigma$ induces an automorphism in the abelian group $C$.

Every $\sigma$ in $\theta$ is an automorphism of $V$ and $\theta$ is a group of automorphisms of $V$ which is essentially the same as the group of all the automorphisms of $C$. Since $C$ is finite, so is $\theta$. The ring of endomorphisms of $V$ which is spanned by $\theta$ contains for every pair $x, y$ of elements in $B$ one and only one linear transformation of $V$ over $A$ which maps $x$ onto $y$ and all the other elements in $B$ upon 0 , since $\theta$ is transitive on the elements, not 0 , in $C$. These linear transformations form a soc. system of matrix units over $A$, showing again that $\theta$ is a finite irreducible group of linear transformations of the vector space $V$ over 4 . Thus we have shown in both cases that (c) is a consequence of (a).

Assume finally the validity of (c). Then there exists a finite irreducible group $\theta$ of linear transformations of the vector space $V$ over $A$. If $v \neq 0$ is an element in $V$, then $v \theta$ is a finite subset of $V$ which spans a subspace $S \neq 0$ of the vector space $V$ over 4 . Since 
$S$ is $\theta$-admissible and $\theta$ is irreducible, we have $S=V$; and from the finiteness of $v \theta$ we deduce the finiteness of the rank of $V$. This completes the proof.

COROLlary 1. The following properties of the irreducible ring $\Lambda$ of endomorphisms of the abelian group $A$ and of the centralizer $\triangle$ of $A$ [within the ring of endomorphisms of $A$ ] are equivalent provided the rank of $A$ over $A$ is $>1$ :

(i) $\Lambda$ is spanned by an almost abelian torsion group of automorphisms of $A$.

(ii) $\Delta$ is a commutative, absolutely algebraic field of prime number characteristic $p$ [so that $p A=0$ ] and the rank of $A$ over $\triangle$ is finite.

(iii) The rank of $A$ over $\triangle$ is finite and the group of automorphisms in $\Lambda$ is locally finite.

(iv) $\Lambda$ is the centralizer of $\Delta$ and the group of automorphisms in $\Lambda$ is locally finite.

Proof. It is the content of our Theorem that (ii) is a consequence of (i).--If (ii) is true, then we note that $\Lambda$ is a ring of linear transformations of the vector space $V$ over $\Delta$; and it is well known [and easily verified] that because of (ii) finite subsets of $\Lambda$ span finite subrings of $A$; cp. Proposition (c). Thus (iii) is a consequence of (ii).

Assume the validity of (iii). Because of the irreducibility of $\Lambda$ we may deduce from Schur's Lemma-see Jacobson [p. 26, Theorem 2]-that $\Delta$ is a [not necessarily commutative] field. Since the rank of $A$ over $\Delta$ is finite, we may apply Lemma 3 . Consequently

$(+) \Lambda$ is the ring of all linear transformations of the vector space $A$ over the field $A$; and

$(++)$ there exists a finite irreducible group $\theta$ of linear transformations of the vector space $A$ over the field $A$.

Denote by $\Gamma$ the group of all the automorphisms in $\Lambda$. Then we deduce from (+) easily [because of the finiteness of the rank] that $\Lambda$ is spanned by $\Gamma$ and that $\Gamma$ is consequently an irreducible group of automorphisms of $A$. Since $\Gamma$ is locally finite [by (iii)], it follows from our Proposition that $\Delta$ is a commutative field. Application of $(+)$ shows that $\Delta$ is the center of $\Lambda$. Another application of $(+)$ shows that the group $\theta$ [of $(++)]$ is contained in $\Lambda$. If $\Delta^{*}$ is the multiplicative group of all the elements, not 0 , in $A$, then $A^{*} \theta$ is a subgroup of $\Gamma$ which is clearly an almost abelian torsion group of automorphisms of $A$. The irreducibility of this group of automorphisms of $A$ is a consequence of $(++)$; and thus we have deduced (i) from (iii). 
If the equivalent conditions (i)-(iii) are satisfied by $A$, then $\Lambda$ is by Lemma 3 the centralizer of $\Delta$ so that (iv) is a consequence of (i) to (iii).-Assume conversely the validity of (iv). If the rank of $A$ over $\Delta$ were infinite, then there would exist a linear transformation $\sigma$ of the vector space $A$ over $\Delta$ which induces a permutation of order 0 on some basis of $A$ over $\Delta$. Clearly $\sigma$ is an automorphism of $A$; and $\sigma$ belongs by (iv) to the group of automorphisms in $\Lambda$. This group is consequently not a torsion group; but, by (iv), it is locally finite. This contradiction shows the finiteness of the rank of $A$ over $\Delta$ so that (iii) is a consequence of (iv), completing the proof.

REMARK 4. The reader should note that we have deduced (i) from (iii) in the following somewhat stronger form:

(i*) $A$ is spanned by a torsion group of automorphisms with finite central quotient group.

Inspection of this proof shows that we have deduced from (iii) the following facts [which are clearly contained in the equivalent conditions (i)-(iv)]:

$\Delta$ is part and hence the center of $A$ and $A$ contains a finite group $\theta$ of automorphisms such that the ring $A$ is spanned by $A, \theta$.

It is then clear that every element in $A$ has the form

$$
\sum_{\sigma \in \theta} \delta(\sigma) \sigma \quad \text { with } \delta(\sigma) \text { in } 4 \text {. }
$$

As $\Lambda$ may be considered as a vector space over its commutative subfield [its center] 4 , we may conclude now that

$$
\Delta \text { has finite rank over its center } \triangle \text {. }
$$

REMark 5. Condition (iii) is contained in conditions (ii) and (iv); and it is an immediate consequence of Lemma 3 that (iv) may be deduced from (iii). Thus (iv) appears somewhat weaker than (iii) and (iii) does not tell anything that it is not contained in the other conditions. Observation of the proofs shows that the insertion of (iii) has been convenient for them.

REMARK 6 . Suppose that $\Delta$ is a commutative, absolutely algebraic field of prime number characteristic $p$, that $V$ is a vector space of finite rank over $\Delta$ and that $A$ is the ring of all the linear transformations of $V$ over $A$. Assume furthermore that $\Gamma$ is a group of automorphisms in $\Lambda$ spanning $\Lambda$. Then $\Gamma$ and $\Lambda$ are irreducible; and it is a consequence of Corollary 1 that

(*) $\quad \Gamma$ is locally finite; 
and we deduce from Lemma 3 the existence of a finite irreducible group $\theta$ of linear transformations of the vector space $V$ over $\Delta$. Since the ring $A$ is spanned by $\Gamma$, and since $\theta$ is finite, there exists a finite subset $\Sigma$ of $\Gamma$ such that $\theta$ is part of the subring $\Sigma$. By $\left({ }^{*}\right)$ a finite subgroup $\bar{\Sigma}$ of $\Gamma$ is generated by $\Sigma$. Since $\Sigma$ and $\bar{\Sigma}$ span the same subring $\Sigma^{*}$ of $\Lambda$ which contains the irreducible group $\theta$ of linear transformations, we have shown:

$\left.{ }^{* *}\right) \quad \Gamma$ contains a finite irreducible group of linear transformations of $V$ over $\Delta$.

If it were known that the elements, not 0 , in $\Delta$ are contained in $\Gamma$, then we would have shown that $\Gamma$ contains an irreducible torsion group of automorphisms of $V$ with finite central quotient group; and this would constitute a considerable improvement on the property $\left(\mathrm{i}^{*}\right)$ of Remark 4. But we have not been able to decide whether or not $\Gamma$ contains an irreducible, almost abelian torsion group of automorphisms of $V$.

CoRollary 2. The following properties of the irreducible, almost abelian torsion group $\Gamma$ of automorphisms of the abelian group $A$ are equivalent:

(i) $A$ is finite.

(ii) $A$ is of finite rank.

(iii) The orders of the elements in $\Gamma$ are bounded.

(iv) $\Gamma$ is finite.

(v) If the abelian torsion group $\theta$ of automorphisms of A centralizes $\Gamma$, then the orders of the elements of $\theta$ are bounded.

Proof. It is fairly obvious that the conditions (ii)-(v) are consequences of the finiteness of $A$. To prove the converse we note first that as a consequence of the Theorem and of the general hypotheses of our Corollary the following properties of $A, \Gamma$ are satisfied:

(1) $A$ is an elementary abelian $p$-group.

(2) The centralizer $\Delta$ of $\Gamma$ within the ring of endomorphisms of $A$ is a commutative, absolutely algebraic field of characteristic $p$.

(3) The rank of $A$ over $\Delta$ is finite.

(4) The rank of $\Gamma$ is finite.

Elementary abelian $p$-groups of finite rank are finite, since the orders of its finite subgroups are bounded. Hence (i) is a consequence of (ii) and (1).

Assume next that the orders of the elements in $\Gamma$ are bounded. There exists by hypothesis an abelian subgroup $\Sigma$ of $\Gamma$ whose index $[\Gamma: \Sigma]$ is finite. The rank of $\Sigma$ is finite by (4) and $\Sigma$ is a torsion group the orders of whose elements are bounded. Then $\Sigma$ is the direct 
product of finitely many primary groups; and the primary components of $\Sigma$ are finite, since they are of finite rank and the orders of their elements are bounded. Hence $\Sigma$ itself is finite, implying the finiteness of $\Gamma$. Thus (iv) is a consequence of (iii).

If $\Gamma$ is finite, then we deduce from (1) that every element in $A$ is contained in a finite $\Gamma$-admissible subgroup of $A$. Thus the finiteness of $A$ is a consequence of the irreducibility of $\Gamma$ and we have verified the equivalence of (i)-(iv).

Assume finally the validity of $(\mathrm{v})$. The elements not 0 in $\Delta$ form by (2) an abelian torsion group $\theta$ of rank 1 which by (v) is finite. Hence $\Delta$ is finite. Application of (3) shows the finiteness of $A$, completing the proof.

COROLlary 3. Assume that $\Delta$ is a commutative, absolutely algebraic field of characteristic $p$ [a prime], that $V$ is a vector space over $\Delta$ and that $\Gamma$ is the group of all [regular] linear transformations of $V$ over 4 . Then the rank of $\Gamma$ is finite if, and only if, $V$ is finite or the rank of $V$ over $A$ is [0 or] 1.

Proof. It is clear that our conditions are sufficient for the finiteness of the rank of $\Gamma$; and thus we assume next that the rank of $\Gamma$ is finite.

Assume first by way of contradiction that the rank of $V$ over $\Delta$ is infinite. Then it is easy to construct a subgroup of $\Gamma$ which induces a group of permutations in some preassigned basis of $V$ over $\Delta$ and which is an infinite, elementary abelian 2-group. This subgroup is not of finite rank so that $\Gamma$ itself is not of finite rank. This is a contradiction showing that

(+) the rank $n$ of $V$ over $\Delta$ is finite.

Assume next that $1<n$. Then we may represent $V$ in the form $V=S \oplus T$ where $S$ and $T$ are subspaces of $V$ over $\Delta$ and where the rank of $S$ is 2. Denote by $a, b$ a basis of $S$. Consider the set $\theta$ of all the linear transformations of $V$ over $\Delta$ with the properties:

$$
\begin{aligned}
\alpha \sigma & =a+\delta b \text { with } \delta \text { in } \Delta, \\
b \sigma & =b \text { and } x \sigma=x \text { for } x \text { in } T .
\end{aligned}
$$

It is easily seen that $\theta$ is a subgroup of $\Delta$ which is isomorphic to the additive group of $\Delta$. Hence $\theta$ is an elementary abelian $p$-group; and the rank of $\theta$ is finite if, and only if, $\theta$ is finite. But the rank of $\Gamma$ is finite implying the finiteness of the rank of the subgroup $\theta$ of $\Gamma$. Consequently $\theta$ is finite; and this implies the finiteness of the field $\Delta$. Application of $(+)$ shows that $V$ is finite; and thus we have shown: 
If $1<n$, then $V$ is finite.

But this is just the fact that we wanted to prove.

REMARK 7. One should compare Corollary 3 and the statement (D) of the Theorem.

\section{BIBLIOGRAPHY}

1. Reinhold Baer, Nilpotent groups and their generalizations, Trans. Amer. Math. Soc., 47 (1940), 393-434.

2. - Groups with descending chain condition for normal subgroups, Duke Math. J., 16 (1949), 1-22.

3. - The hypercenter of a group, Acta Math., 89 (1953), 165-208.

4. — Gruppentheoretische Eigenschaften, Math. Ann., 149 (1963), 181-210.

5. A. M. Duguid and D. H. McLain, $F C$-nilpotent and $F C$-soluble groups, Proc. Cambridge Phil. Soc., 52 (1955), 391-398.

6. N. Jacobson, Structure of Rings, Amer. Math. Soc. Coll. Publ. 37 (1956).

7. Wilhelm Specht, Gruppentheorie, Berlin-Göttingen-Heidelberg 1956.

Mathematisches Seminar Der

UNIVERSITÄT FRANKFURT A.M. 


\title{
PACIFIC JOURNAL OF MATHEMATICS
}

\author{
EDITORS
}

\author{
Robert Osserman \\ Stanford University \\ Stanford, California \\ M. G. Arsove \\ University of Washington \\ Seattle 5, Washington
}

\author{
J. Dugundji \\ University of Southern California \\ Los Angeles 7, California \\ Lowell J. Paige \\ University of California \\ Los Angeles 24, California
}

\section{ASSOCIATE EDITORS}
E. F. BECKENBACH
B. H. NEUMANN
F. WOLF
K. YOSHIDA

\section{SUPPORTING INSTITUTIONS}

\author{
UNIVERSITY OF BRITISH COLUMBIA \\ CALIFORNIA INSTITUTE OF TECHNOLOGY \\ UNIVERSITY OF CALIFORNIA \\ MONTANA STATE UNIVERSITY \\ UNIVERSITY OF NEVADA \\ NEW MEXICO STATE UNIVERSITY \\ OREGON STATE UNIVERSITY \\ UNIVERSITY OF OREGON \\ OSAKA UNIVERSITY \\ UNIVERSITY OF SOUTHERN CALIFORNIA
}

\author{
STANFORD UNIVERSITY \\ UNIVERSITY OF TOKYO \\ UNIVERSITY OF UTAH \\ WASHINGTON STATE UNIVERSITY \\ UNIVERSITY OF WASHINGTON \\ AMERICAN MATHEMATICAL SOCIETY \\ CALIFORNIA RESEARCH CORPORATION \\ SPACE TECHNOLOGY LABORATORIES \\ NAVAL ORDNANCE TEST STATION
}

Mathematical papers intended for publication in the Pacific Journal of Mathematics should by typewritten (double spaced), and on submission, must be accompanied by a separate author's résumé. Manuscripts may be sent to any one of the four editors. All other communications to the editors should be addressed to the managing editor, L. J. Paige at the University of California, Los Angeles 24, California.

50 reprints per author of each article are furnished free of charge; additional copies may be obtained at cost in multiples of 50 .

The Pacific Journal of Mathematics is published quarterly, in March, June, September, and December. Effective with Volume 13 the price per volume (4 numbers) is $\$ 18.00$; single issues, $\$ 5.00$. Special price for current issues to individual faculty members of supporting institutions and to individual members of the American Mathematical Society: $\$ 8.00$ per volume; single issues $\$ 2.50$. Back numbers are available.

Subscriptions, orders for back numbers, and changes of address should be sent to Pacific Journal of Mathematics, 103 Highland Boulevard, Berkeley 8, California.

Printed at Kokusai Bunken Insatsusha (International Academic Printing Co., Ltd.), No. 6, 2-chome, Fujimi-cho, Chiyoda-ku, Tokyo, Japan.

PUBLISHED BY PACIFIC JOURNAL OF MATHEMATICS, A NON-PROFIT CORPORATION

The Supporting Institutions listed above contribute to the cost of publication of this Journal, but they are not owners or publishers and have no responsibility for its content or policies. 


\section{Pacific Journal of Mathematics}

\section{Vol. 14, No. $2 \quad$ June, 1964}

Tom M. (Mike) Apostol and Herbert S. Zuckerman, On the functional equation $F(m n) F((m, n))=F(m) F(n) f((m, n)) \ldots \ldots \ldots \ldots \ldots \ldots \ldots \ldots \ldots \ldots \ldots$

Reinhold Baer, Irreducible groups of automorphisms of abelian groups . . . . . . . 385

Herbert Stanley Bear, Jr., An abstract potential theory with continuous kernel . . . . 407

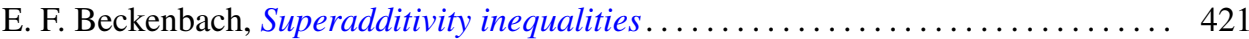

R. H. Bing, The simple connectivity of the sum of two disks . . . . . . . . . . . 439

Herbert Busemann, Length-preserving maps ...................... 457

Heron S. Collins, Characterizations of convolution semigroups of measures . . . . . . 479

Paul F. Conrad, The relationship between the radical of a lattice-ordered group and complete distributivity............................ 493

P. H. Doyle, III, A sufficient condition that an arc in $S^{n}$ be cellular . . . . . . . . . 501

Carl Clifton Faith and Yuzo Utumi, Intrinsic extensions of rings . . . . . . . . . . 505

Watson Bryan Fulks, An approximate Gauss mean value theorem . . . . . . . . . . 513

Arshag Berge Hajian, Strongly recurrent transformations . . . . . . . . . . . . . 517

Morisuke Hasumi and T. P. Srinivasan, Doubly invariant subspaces. II . . . . . . . 525

Lowell A. Hinrichs, Ivan Niven and Charles L. Vanden Eynden, Fields defined by

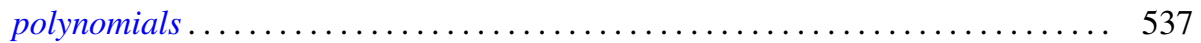

Walter Ball Laffer, I and Henry B. Mann, Decomposition of sets of group

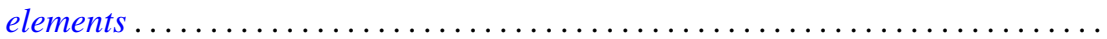

John Albert Lindberg, Jr., Algebraic extensions of commutative Banach

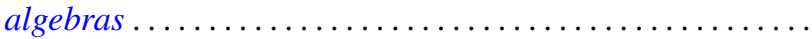

W. Ljunggren, On the Diophantine equation $C x^{2}+D=y^{n} \ldots$

M. Donald MacLaren, Atomic orthocomplemented lattices ....

Moshe Marcus, Transformations of domains in the plane and applications in the

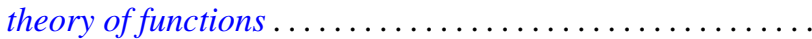

Philip Miles, $B^{*}$ algebra unit ball extremal points . .................. 627

W. F. Newns, On the difference and sum of a basic set of polynomials . . . . . . . 639

Barbara Osofsky, Rings all of whose finitely generated modules are injective ...... 645

Calvin R. Putnam, Toeplitz, matrices and invertibility of Hankel matrices . . . . . . . 651

Shoichiro Sakai, Weakly compact operators on operator algebras . . . . . . . . . 659

James E. Simpson, Nilpotency and spectral operators . . . . . . . . . . . . . 665

Walter Laws Smith, On the elementary renewal theorem for non-identically

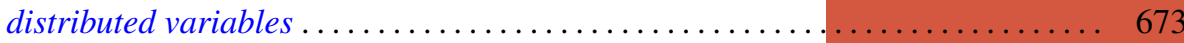

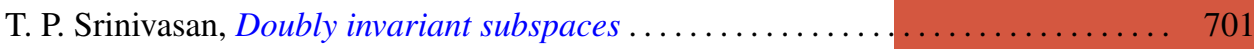

J. Roger Teller, On the extensions of lattice-ordered groups . . . . . . . . . . . . 709

Robert Charles Thompson, Unimodular group matrices with rational integers as

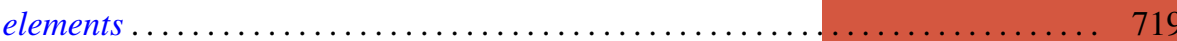

J. L. Walsh and Ambikeshwar Sharma, Least squares and interpolation in roots of unity

Charles Edward Watts, A Jordan-Hölder theorem .................... 731

Kung-Wei Yang, On some finite groups and their cohomology .............. 735

Adil Mohamed Yaqub, On the ring-logic character of certain rings ............ 741

Paul Ruel Young, A note on pseudo-creative sets and cylinders 